\title{
Diseño e integración de un sistema modular para producción de hortalizas, peces y aves de corral, autosustentable
}

\section{Design and integration of a modular system to produce vegetables, fish and poultry, self-sustaining}

CASTILLO-QUIROZ, Gregorio $\dagger^{*}$, ROJAS-BALBUENA, Dorian, LIMON-DIAZ, Miguel Ángel y CRUZ-GARRIDO, Arnulfo

Instituto Tecnológico Superior de Huauchinango, División de Ingeniería Mecatrónica y Maestría en Tecnologías de la Información. México.

ID $1^{\text {er }}$ Autor: Gregorio, Castillo-Quiroz / ORC ID: 0000-0002-1904-4172, Researcher ID Thomson: H-9402-2018, arXiv Author ID: 0000-0002-1904-4172, CVU CONACYT ID: 162009

ID $1^{\text {er }}$ Coautor: Dorian, Rojas-Balbuena / ORC ID: 0000-0002-4770-8669, Researcher ID Thomson: AAN-1530-2020, arXiv Author ID: 0000-0002-4770-8669, CVU CONACYT ID: 299726

ID $2^{\text {do }}$ Coautor: Miguel Ángel, Limon-Diaz / ORC ID: 0000-0002-7578-7077, Researcher ID Thomson: T-6486-2017, arXiv Author ID: 0000-0002-7578-7077, CVU CONACYT ID: 349952

ID $3^{\text {er }}$ Coautor: Arnulfo, Cruz-Garrido / ORC ID: 0000-0001-9366-8525, Researcher ID Thomson: F-8400-2019, arXiv Author ID: 0000-0001-9366-8525, CVU CONACYT ID: 320172

DOI: $10.35429 / J O T I .2020 .13 .4 .1 .11$

Recibido 10 Enero, 2020; Aceptado 30 Junio, 2020

\section{Resumen}

Hoy en día, México enfrenta una crisis por la escasez y el consecuente incremento de los precios en los productos básicos. Se prevé que esta situación continúe, porque la superficie disponible para el cultivo, ganadería y pesca está llegando a su límite, mientras que la población y sus necesidades de alimentación aumentan. El sistema propuesto, presenta una alternativa en la producción de alimentos para el autoconsumo en zonas rurales y urbanas, que consta de tres niveles para crianza de peces, aves de corral y producción de hortalizas, e incorpora la tecnología para poder controlar su desarrollo. El prototipo propuesto contempla una parte estructural que soporta tres módulos. El primer módulo está destinado a la crianza de peces con un sistema de filtraje y recirculación del agua. El segundo módulo es destinado para el cultivo de hortalizas, con un sistema para el control de los nutrientes necesarios de los vegetales, y a su vez, los desechos de estos sirvan de alimento a las aves de corral. El tercer módulo está destinado para la crianza de aves de corral, contando con un sistema que concentra y trata los desechos, que sirven como nutrientes para las hortalizas.
Abstract

Today, Mexico is facing a crisis over scarcity and the consequent rise in commodity prices. This situation is expected to continue, because the area available for cultivation, livestock and fishing is reaching its limit, while the population and its food needs are increasing. The proposed system presents an alternative in the production of food for self-consumption in rural and urban areas, consisting of three levels for fish rearing, poultry and vegetable production, and incorporates technology to control its development. The proposed prototype envisages a structural part that supports three modules. The first module is intended for the rearing of fish with a system of filtration and recirculation of water. The second module is intended for the cultivation of vegetables, with a system for the control of the necessary nutrients of vegetables, and in turn, the wastes of plants serve as food to poultry. The third module is intended for poultry rearing, with a system that concentrates and treats waste, which serve as nutrients for vegetables.

Autosustentable, Sistema, Producción

Self-sustainability, System, Production

Citación: CASTILLO-QUIROZ, Gregorio, ROJAS-BALBUENA, Dorian, LIMON-DIAZ, Miguel Ángel y CRUZGARRIDO, Arnulfo. Diseño e integración de un sistema modular para producción de hortalizas, peces y aves de corral, autosustentable. Revista de Invención Técnica. 2020. 4-13:1-11.

*Correspondencia al Autor (Correo Electrónico: gcquiroz1977@gmail.com)

$\dagger$ Investigador contribuyendo como primer autor. 


\section{Introducción}

El sector alimentario y el sector agrícola brindan soluciones claves para el desarrollo de un país y son vitales para la eliminación del hambre y la pobreza. Actualmente, nuestros suelos, ríos, lagos, arroyos, mares y nuestra biodiversidad están padeciendo un rápido proceso de degradación debido a procesos de sobreexplotación, cambio climático, desastres naturales, crisis económicos, conflictos y desplazamiento de población.

Diversos gobiernos y organismos multinacionales han hecho hincapié en la reducción del hambre y la desnutrición, dado que, según declaraciones de la Organización de las Naciones Unidas para la Agricultura y la Alimentación (FAO), el problema de la desnutrición por deficiencia en el consumo radica en el acceso desigual a los alimentos.

En este contexto, el Consejo Nacional de Evaluación de la Política de Desarrollo Social (CONEVAL), en el caso de México, según datos del mismo organismo la pobreza extrema alimentaria afecta al $18.2 \%$ de la población, otro dato inquietante es que el porcentaje de población que sufre hambre y desnutrición en el ámbito mundial es del 50\%.

México es un país que está constantemente en desarrollo y la tecnología poco a poco se va adentrando en el sector alimentario y agrícola. Pues como afirma el autor Durán (2014), la tecnología es un factor de desarrollo tecnológico en los diferentes sectores de la comunidad.

Hoy en día conseguir vegetales sanos, libre de pesticidas es una preocupación que ha llevado a muchos organismos a buscar las mejores soluciones para evitar enfermedades. Una alternativa de producción de alimentos es la agricultura vertical. Un huerto vertical es una estructura que está compuesta por plantas; permite plantar frutas o verduras para el consumo diario. Para su implementación no se requiere de un terreno, sino de un espacio por ejemplo una pared, donde reciba la suficiente luz y aire durante el día. En cambio, un huerto tradicional es considerado como un espacio que se encuentra cerca de un domicilio, en donde crecen arbustos, árboles y herbáceas silvestres y demás cultivos y animales que generalmente no ocupan mucho espacio (Cano, 2015).
El objetivo del presente proyecto es diseñar e implementar un prototipo modular autosustentable vertical para crianza de peces, aves de corral y hortalizas, orientada a la seguridad alimentaria en la región de la Sierra Norte de Puebla. La propuesta contempla una parte estructural y de soporte para tres niveles, los cuales son módulos desmontables para que se facilite la producción en serie, el transporte, el montaje, el suministro e inclusive la posible solución de problemas.

El primer módulo está destinado a la crianza de peces con un sistema de filtraje y recirculación del agua. El segundo módulo está destinado a la producción de hortalizas de la región, con un sistema para el control de los nutrientes necesarios de los vegetales, y a su vez, los desechos de estos sirven de alimento a las aves de corral. El tercer módulo es para la crianza de aves de corral, en el cual se implementó un sistema mecánico que concentra y trata los desechos, que sirven como nutrientes para las hortalizas.

Derivado del diseño del sistema permite la disponibilidad de alimentos en traspatio reduciendo gastos en las familias de escasos recursos. Sabemos que la alimentación está íntimamente relacionada con las condiciones de salud de las personas, por lo que el proyecto en cierta medida aporta a este problema. Orientado a la seguridad alimentaria en zonas de escasos recursos, sobre todo en el sector rural que padecen hambre: Las mujeres en proceso de gestación, estudios indican que durante la etapa de gestación se presenta un incremento de las necesidades de nutrientes, las mujeres en estado de desnutrición durante el embarazo tienen mayor riesgo de parto prematuro, bajo peso del bebé al nacer e incluso, una mayor probabilidad de sufrir un aborto espontáneo.

Los adultos mayores estudios indican que la desnutrición en ancianos está relacionada con múltiples consecuencias, que incluyen: alteración en la función muscular, disminución de la masa ósea, disfunción inmunitaria, anemia, repercusión en el estado cognitivo, pobre cicatrización, pobre recuperación posterior a una cirugía, incremento del riesgo de institucionalización, mayor estancia hospitalaria, fragilidad, mortalidad y reducción en la calidad de vida. 
Los niños y adolescentes para desarrollar y mantener una buena salud, dado que en la adolescencia existen necesidades energéticas, proteicas y de micronutrientes que supera cualquier otra época de la vida. Es bien sabido que, en la pubertad se adquiere el 25 por ciento de la talla adulta, se aumenta un 50 por ciento la masa esquelética, se duplica la masa muscular (sobre todo en el sexo masculino) y se produce un aumento del volumen sanguíneo y de los órganos internos. Todos estos cambios, tan importantes para el desarrollo y la correcta formación de una persona adulta, para garantizar un correcto desarrollo físico y psicológico.

De esta manera el proyecto pretende que a partir de la metodología puesta en marcha pueda ser implementada en cualquier región del País. El desarrollo de este trabajo de investigación está dividido en las secciones que a continuación se describen:

\section{Metodología}

Se describen los pasos que se siguieron para el desarrollo de la investigación.

\section{- $\quad$ Resultados}

En esta sección se analizan los resultados obtenidos para determinar si se ha logrado el objetivo.

\section{Agradecimientos}

Se agradece a las personas e instituciones que permitieron el desarrollo de esta investigación.

\section{Conclusiones}

Se habla de los objetivos logrados satisfactoriamente y de cómo contribuye este trabajo en diversas aplicaciones relacionadas con la producción de hortalizas, aves de corral y peces.

\section{Metodología}

El sistema modular autosustentable (ver Figura 1), se implementó buscando la necesidad de garantizar el suministro continuo de alimentos dentro de tres ejes principales, aves de corral, hortalizas y peces.
El primer módulo está destinado a la crianza de peces con un sistema de filtraje y recirculación del agua. El segundo módulo está destinado a la producción de hortalizas, con un sistema para el control de los nutrientes necesarios de los vegetales, y a su vez, los desechos de estos sirvan de alimento a las aves de corral. El tercer módulo contempla la crianza de aves de corral, contando con un sistema que concentra y trata los desechos, que sirven como nutrientes para las hortalizas. Otra característica, es que el sistema provee de los nutrientes necesarios utilizando un lazo cerrado simbiótico, permitiendo producir productos totalmente orgánicos, colaborando en favor del medio ambiente, disminuyendo el uso de herbicidas, fungicidas y de agroquímicos.

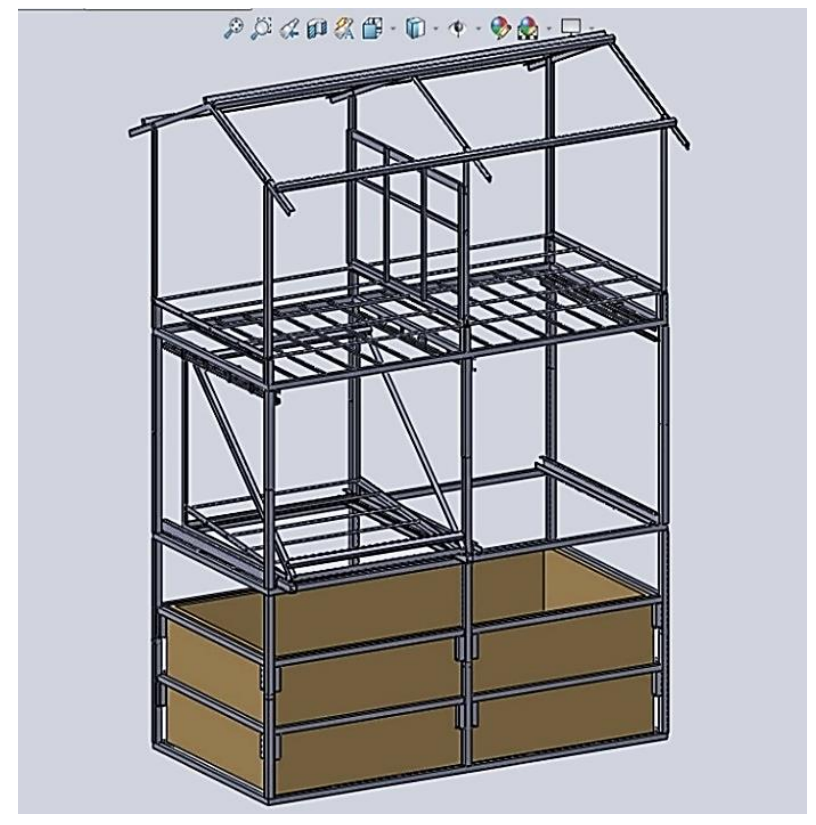

Figura 1 Bosquejos del prototipo

Fuente: Elaboración propia

\section{Filosofía de operación}

En el primer nivel se adapta un contenedor de agua para criar peces, tiene una capacidad de soportar un volumen total de 1003 litros o 1.003 metros cúbicos que es monitoreado y controlado según sean las necesidades, de los cuales el $75 \%$ es ocupado por agua y el resto del volumen es ocupado por los peces hasta ocupar un nivel del 95\% para evitar desbordes de agua. 
El segundo nivel es para el sistema hidropónico destinado para el cultivo de hortalizas de hojas, las paredes están forradas de un material ligero que mantiene la temperatura dentro de la estructura, el cual está dividido en dos secciones independientes, donde cada sección cuenta con un cajón deslizable hacia enfrente y hacia atrás para que el operador tenga libertad de maniobrar cuando se requiera, estos cajones son la base para la tierra para plantar las hortalizas.

En el tercer nivel es una nave para la crianza de aves de corral, posee las siguientes medidas de $1 \mathrm{~m}$ de ancho, $2 \mathrm{~m}$ de largo y $0.7 \mathrm{~m}$ de altura, sus paredes son de bajo peso para facilitar su extracción cuando sea necesario desmontarlo, cuenta con un piso de malla de alambre de $2 \mathrm{~cm}$ de diámetro que permita la caída de las heces de las aves a una lámina de acero para la concentración de los desechos para ser trasladados a contenedor para su almacenamiento, para que no exista derrame cerca de la estructura.

\section{Diseño del sistema modular}

Los requerimientos para el diseño del prototipo autosustentable vertical se desarrollaron en el software SolidWorks con las dimensiones reales: El primer nivel está destinado a la crianza de peces, el cual debe contar con una estructura metálica que tenga soportes en los costados y en la base principal, donde los peces deberán desarrollarse, posteriormente se diseñó una estructura capaz de soportar el peso del tanque con su contenido, como se muestra en la Figura 2. Dicho contenedor deberá diseñarse con un sistema de filtraje, recirculación, censado de $\mathrm{PH}$, un sistema de calentamiento y oxigenación del agua, véase Figura 3.

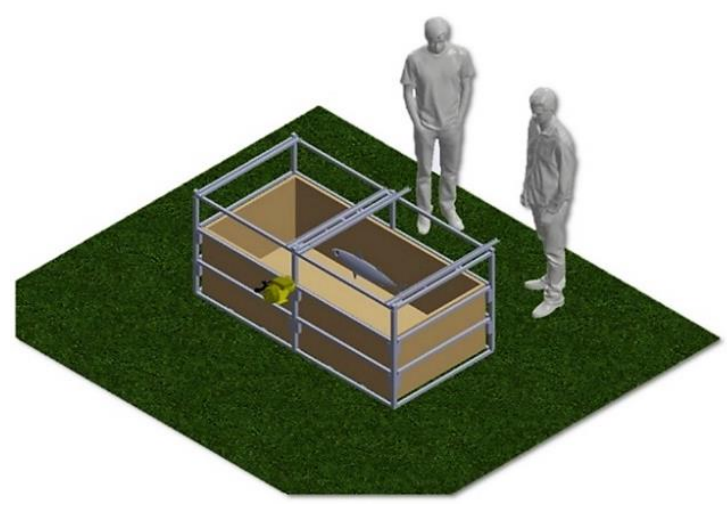

Figura 2 Primer Nivel - Crianza de peces Fuente: Elaboración propia

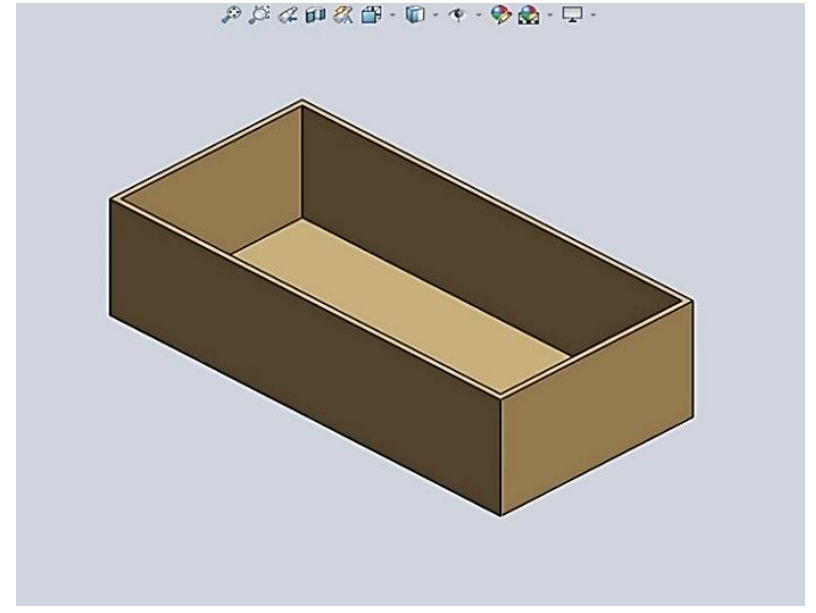

Figura 3 Contenedor de agua

Fuente: Elaboración propia

El segundo nivel está destinado a la producción de hortalizas, dividido en dos cajones estructurales separados e inclinados a $15^{\circ}$ como se muestra en la Figura 4 , los cuales cuentan con un riel que les permite el desplazamiento al frente y atrás, con el objetivo de proporcionar espacio al operador en caso de maniobra.

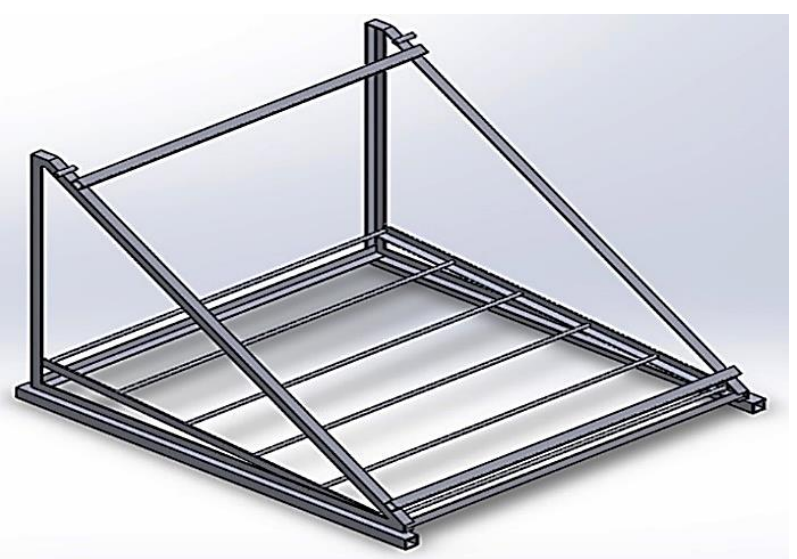

Figura 4 Estructura intermedia del segundo nivel Fuente: Elaboración propia

Además cuenta con un sistema de riego, el cual está habilitado por medio de un circuito de tubería de PVC que proporciona la oxigenación y dosificación de nutrientes, véase Figura 5.

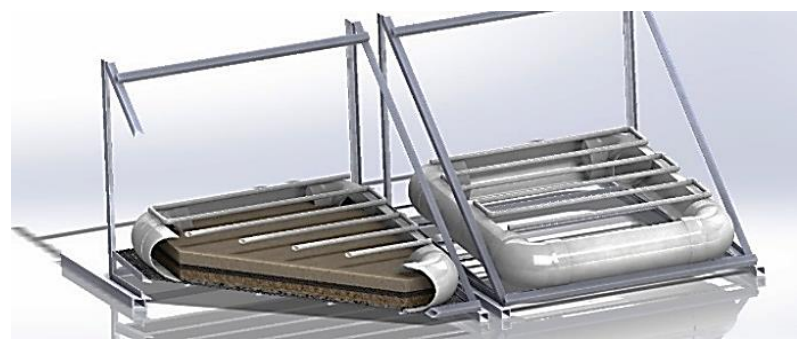

Figura 5 Segundo Nivel - Producción de hortalizas Fuente: Elaboración propia 
En la Figura 6, se muestra el diseño del tercer nivel destinado a la crianza de aves de corral, el cual cuenta con una estructura de soporte con perfil tubular cuadrado y angular, con un techo de lámina a dos aguas, además se implementó un sistema mecánico para la concentración de los desechos, un sistema de calefacción, un sistema de oxigenación, y un sistema de extracción de gas amoniaco, que cumple las funciones de evitar enfermedades de vías respiratorias en las aves.

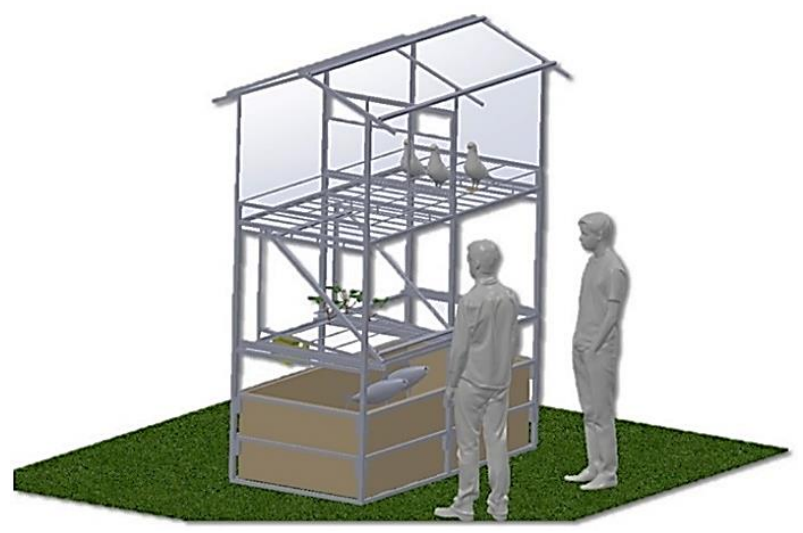

Figura 6 Tercer Nivel - Crianza de aves de corral Fuente: Elaboración propia

Posterior al dimensionamiento de la estructura del galpón avícola se llevó a cabo el diseño general de las partes del tercer nivel, véase Figura 7.

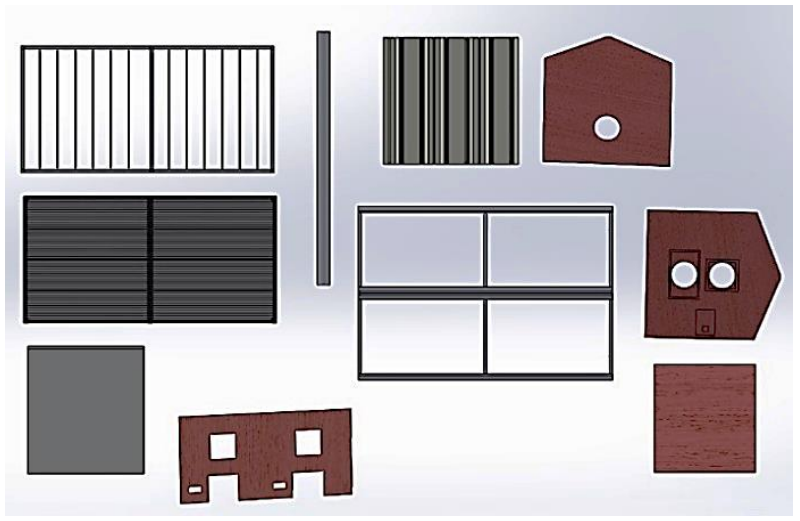

Figura 7 Piezas estructurales del tercer Nivel Fuente: Elaboración propia

Tomando en cuenta las medidas necesarias de cada pieza, se realizó un ensamble final como el que podemos observar en la Figura 8 , la cual muestra de manera previa como fueron ubicados cada uno de los elementos.

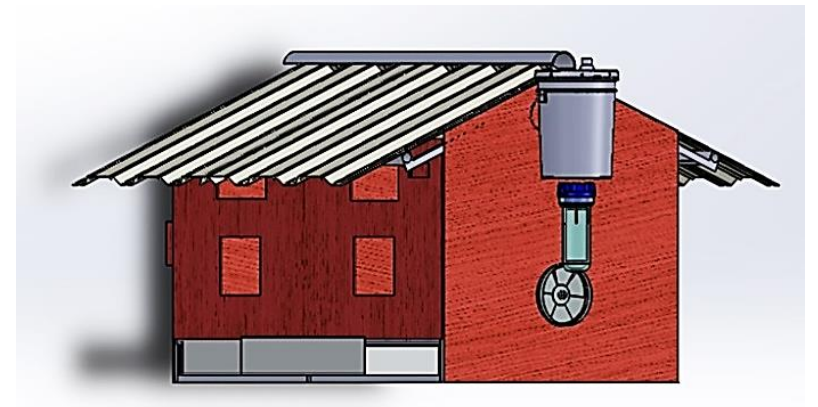

Figura 8 Ensamble final del tercer nivel Fuente: Elaboración propia

\section{Propuesta de tecnologías de control y de automatización}

La automatización de actividades productivas mediante soluciones tecnológicas es una apuesta clara para la disminución en costos de producción y un mayor control de los parámetros de calidad y seguridad además de que las características del sector son idóneas para aplicar, sobre todo por la precisión en el control de variables específicas, la elevada necesidad de mano de obra calificada y entrenada y la repercusión sobre parámetros de calidad e higiene.

La capacidad de producir alimentos vegetales y animales depende en gran medida del clima: la temperatura, la luz y el agua. Las fluctuaciones a corto y a largo plazo de las pautas del clima pueden tener repercusiones extremas en la producción agrícola, y hacer que se reduzca drásticamente el rendimiento de las cosechas, lo que obliga a utilizar nuevas prácticas agrícolas en respuesta a la modificación de las condiciones. Por esta razón, la automatización en esta área es una opción viable.

Partiendo con el análisis mediante datos obtenidos por las gráficas mostradas en las Figuras 9-12 del comportamiento de las principales variables como son la temperatura, humedad relativa y la luz solar a controlar durante el año, se proponen los algoritmos de control según los valores de referencia para la crianza de aves, peces y cultivo de hortalizas. 


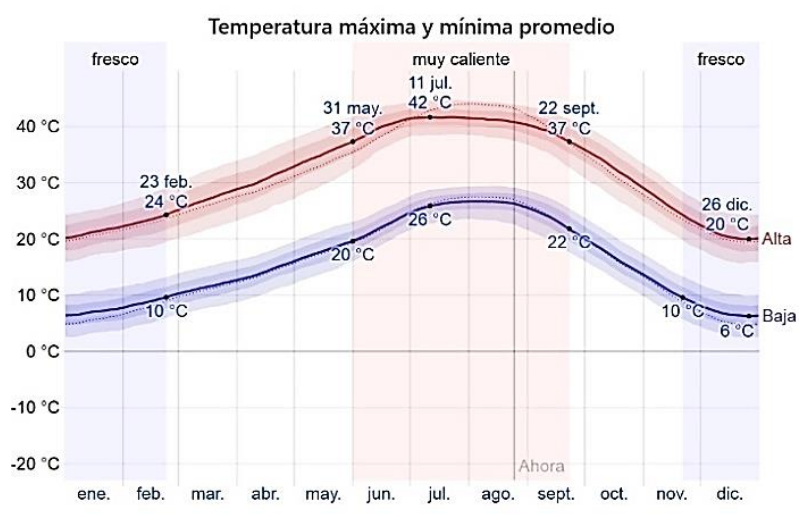

Temperatura promedio por hora

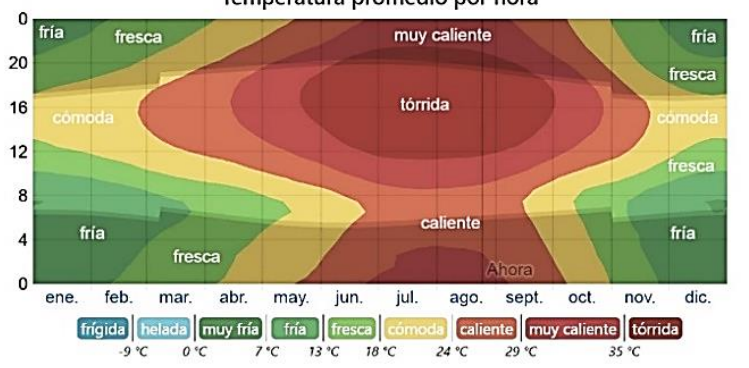

Figura 9 Comportamiento de temperatura durante el año en el Estado de Puebla

Fuente: https://es.weatherspark.com/y/2214/Climapromedio-en-Puebla-M\%C3\%A9xico-durante-todo-ela\%C3\%Blo\#Sections-Clouds

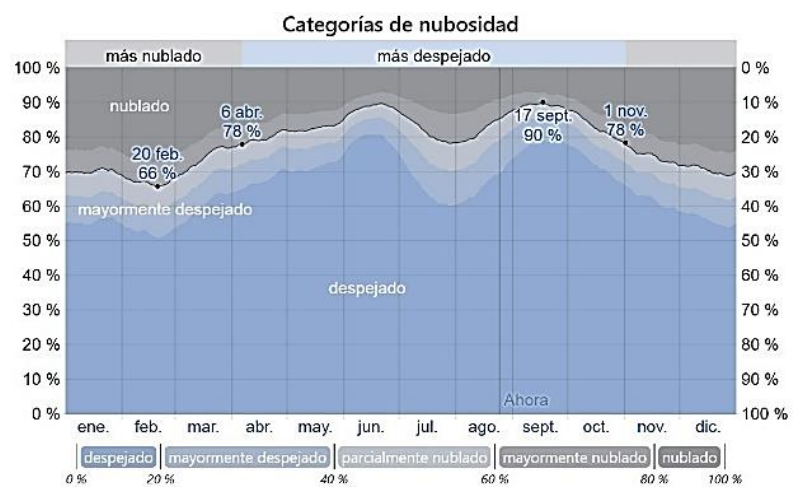

Figura 10 Comportamiento de la nubosidad durante el año en el Estado de Puebla

Fuente: $\quad$ https://es.weatherspark.com/y/2214/Climapromedio-en-Puebla-M\%C3\%A9xico-durante-todo-ela\%C3\%Blo\#Sections-Clouds
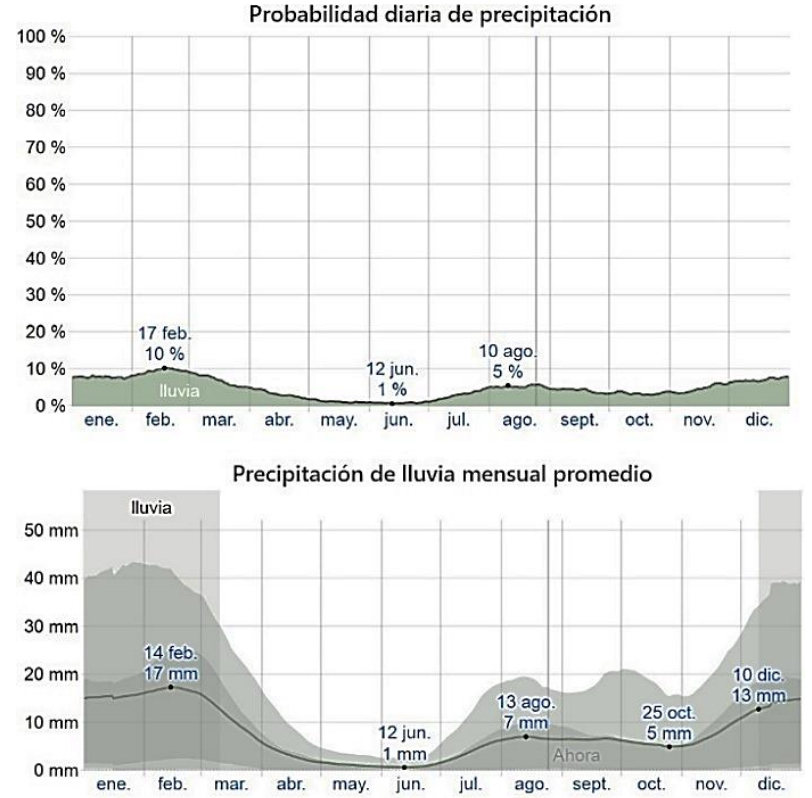

Figura 11 Comportamiento de la precipitación durante el año en el Estado de Puebla

Fuente: $\quad$ https://es.weatherspark.com/y/2214/Climapromedio-en-Puebla-M\%C3\%A9xico-durante-todo-ela\%C3\%B1o\#Sections-Clouds

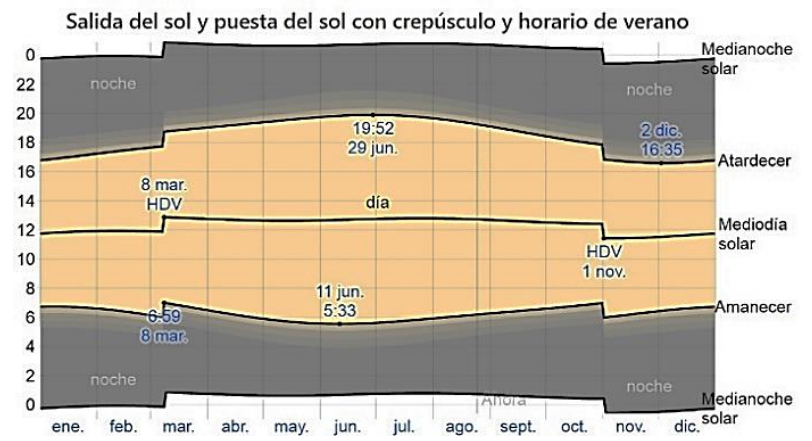

Figura 12 Comportamiento salida y puesta de sol con crepúsculo y horario de verano durante el año en el Estado de Puebla

Fuente: $\quad$ https://es.weatherspark.com/y/2214/Climapromedio-en-Puebla-M\%C3\%A9xico-durante-todo-ela\%C3\%B1o\#Sections-Clouds

El análisis e interpretación de las variables mencionadas permite tener un referente de diseño, ensamble, revestimiento y colocación física del prototipo.

También sirve para dimensionar $\mathrm{y}$ seleccionar el tipo de control a utilizar, los sensores y actuadores, así como, para la selección de valores de ajuste en los algoritmos de control y diseño de la interfaz de monitoreo, graficación y control de los tres módulos y sus alarmas de proceso. 
Particularmente el módulo del tercer nivel se debe censar la temperatura y la humedad relativa para controlar el sistema de calefacción, oxigenación, y extracción de gas amoniaco, cuya finalidad evitar enfermedades de vías respiratorias en las aves, teniendo como referente los siguientes datos proporcionados por los manuales de avicultura:

\begin{tabular}{|r|r|}
\hline Edad en días & Temperatura \\
\hline $1-7$ & $28-32^{\circ} \mathrm{C}$ \\
\hline $8-14$ & $26-28^{\circ} \mathrm{C}$ \\
\hline $15-21$ & $24-26^{\circ} \mathrm{C}$ \\
\hline $22-28$ & $22-25^{\circ} \mathrm{C}$ \\
\hline $29-35$ & $20-22^{\circ} \mathrm{C}$ \\
\hline 36 al sacrificio & $20-22^{\circ} \mathrm{C}$ \\
\hline
\end{tabular}

Tabla 1 Temperatura ideal para aves de corral Fuente: https://avicultura.info/medicion-de-parametrosproductivos-en-avicultural

Uno de los algoritmos a utilizar es el controlador PID, dado que controla con precisión la temperatura en dos modos de operación automática o manual, por lo que se debe de configurar los parámetros para el trabajo en modo automático, en modo manual es necesaria la presencia del operador, el cual se encarga de cerrar el lazo de control. Una vez configuradas las entradas de temperatura y humedad, en una pantalla se observan los dos datos, denominado la Variable de Proceso (VP), en este caso son dos: Humedad Relativa (RH) y Temperatura (T), además del punto de Ajuste (SP).

La variable medida es comparada contra la variable a controlar y el resultado de esta operación es guardada en un registro interno del controlador, posteriormente se le aplica un algoritmo matemático derivado del análisis del proceso a controlar observando tres bandas de comportamiento, siempre dependiente de los límites, una banda proporcional donde el comportamiento es lineal, una vez llegando a su límite actúa la banda proporcional, con la finalidad de acercarse al valor verdadero (SP) de manera progresiva hasta alcanzar la estabilidad, una vez que esta se logró, a cualquier desviación se le aplica la banda derivada, obteniendo así un control puntual de las variables que a su vez activan o desactivan las salidas incrementando o disminuyendo el ancho del pulso.

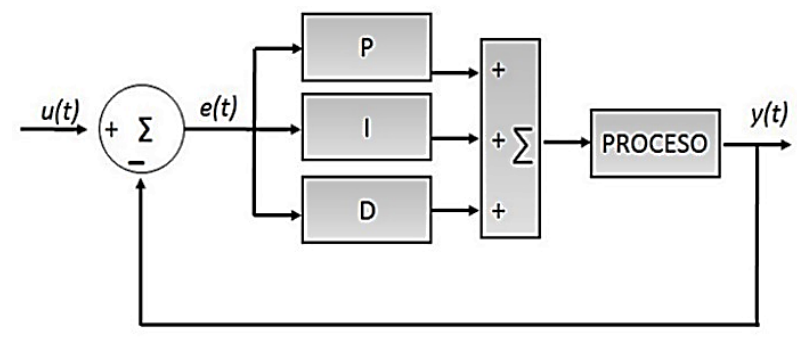

Figura 13 Esquema de control en lazo cerrado Fuente:http://www.ecorfan.org/republicofperu/research_ journals/Revista_de_Ingenieria_Electrica/vollnum3/EC ORFAN_Revista_de_Ingenier\%C3\%ADa_El\%C3\%A9ctr ica_VI_N3_3.pdf

\section{Integración del sistema modular para producción de hortalizas, peces $y$ aves de corral, autosustentable}

En las Figuras 14 y 15 se muestra la manufactura del primer módulo destinado a la crianza de peces, se desarrolló un contenedor para mantener la temperatura estable, siempre dependiente de la especie a trabajar, también se debe mantener el oxígeno suficientemente controlado y cumplir con el ciclo de carbono para el mejor rendimiento y desarrollo de las especies a trabajar, cabe mencionar que se debes estar sensando el PH del agua para el control de los sistemas de filtrado y retroalimentación.

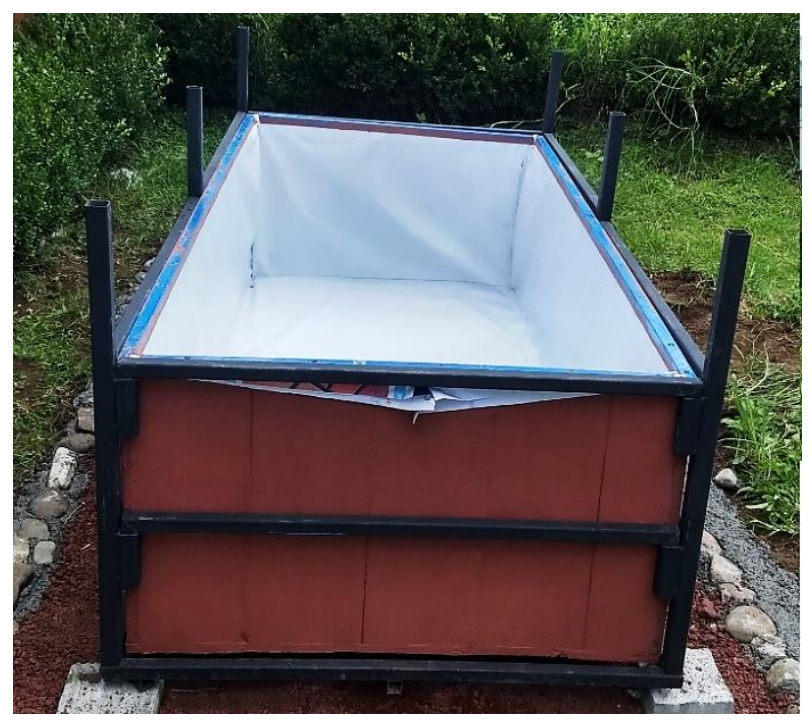

Figura 14 Primer Nivel-Peces

Fuente: Elaboración propia 


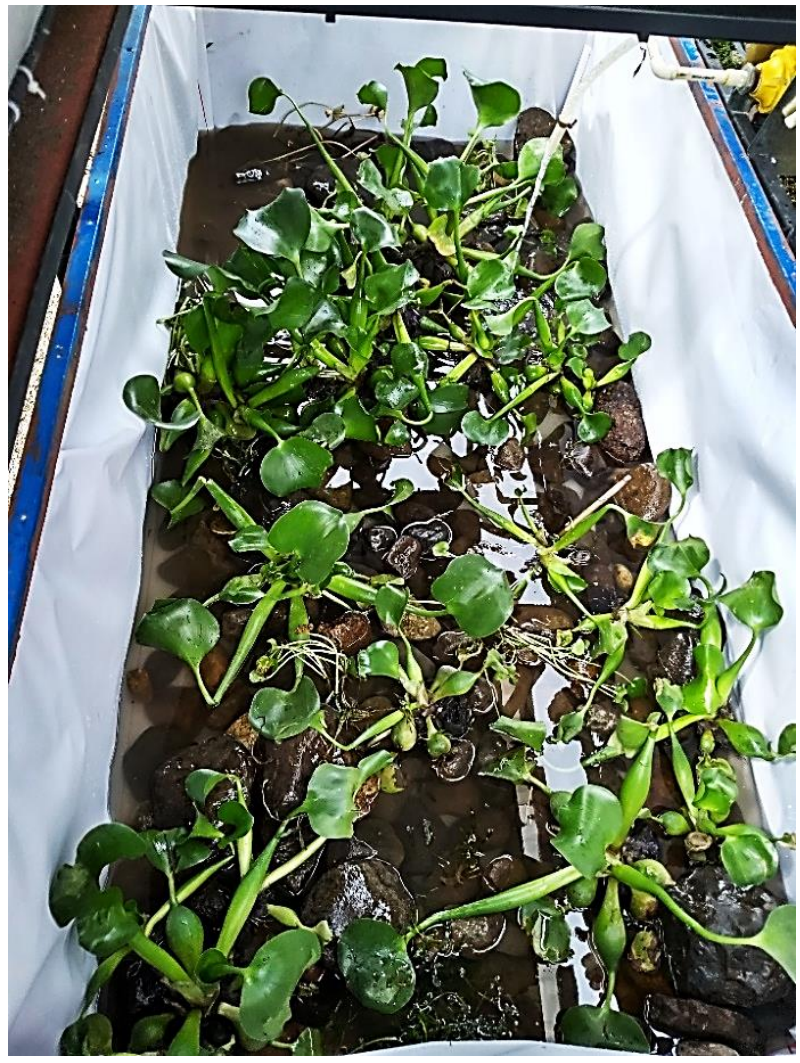

Figura 15 Adecuación del contenedor para el primer nivel Fuente: Elaboración propia

Para el óptimo desarrollo de la tilapia se requiere mantener una temperatura entre $20^{\circ} \mathrm{C}$ a $30^{\circ} \mathrm{C}$, aunque pueden soportar temperaturas menores hasta de $15^{\circ} \mathrm{C}$, que afectan su crecimiento y desarrollo. Por otro lado, la temperatura máxima que soportan se encuentra entre $\operatorname{los} 37-42^{\circ} \mathrm{C}$, que de igual manera afectan al desarrollo.

Además, las concentraciones de oxígeno disuelto: debe controlarse en su valor mínimo de $1 \mathrm{mg} / \mathrm{l}$, aunque lo conveniente es de 2 o $3 \mathrm{mg} / \mathrm{l}$, en particular si no hay mucha luz natural y el $\mathrm{pH}$ a controlar oscila entre 7 y 8, sabiendo que valores menores pueden afectar negativamente al desarrollo e inclusive matar a los peces por otro lado pueden resistir valores alcalinos hasta de 11. La turbidez por mantener es de 30 centímetros de visibilidad.

En la Figura 16 se muestra la manufactura del segundo módulo, el cual contiene dos cajones estructurales de soporte a $45^{\circ}$, los cuales se deslizan hacia enfrente y atrás, con el objetivo de proporcionar espacio al operador en caso de maniobra; un circuito de tubería de PVC que proporcione la posibilidad de hacer fluir el agua con los nutrientes necesarios para el desarrollo de los vegetales.

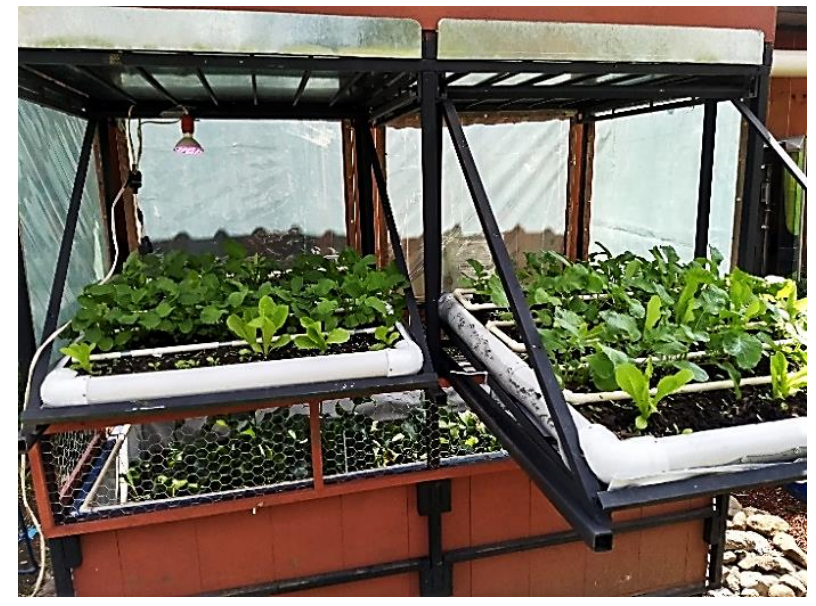

Figura 16 Segundo Nivel - Hortalizas

Fuente: Elaboración propia

El agua utilizada se obtiene del primer módulo, dedicado a la crianza de peces antes de ser filtrada para proporcionar nutrientes derivados de los desechos de los peces y que permite un desarrollo de la planta, sin utilizar fertilizante químico, el desecho de los pollos se recolecta, desecan y en cada nueva siembra se adhiere a la tierra este nutriente 30 partes por 40 partes de tierra negra, 10 partes de gravilla y el resto es de ceniza. Cabe mencionar que los desechos obtenidos de la producción de hortalizas son triturados, los cuales sirven como alimento para los pollos cerrando el ciclo de producción.

El desarrollo del tercer módulo se basó en la estructura de un galpón convencional y una vez revisado el diseño previo se procedió al acondicionamiento físico. Para el techado se utilizó lámina traslucida para aprovechar la luz del día y proporcionar calor dentro del galpón evitando la humedad como podemos observar en la Figura 17, dicha lámina fue colocada con una posición a dos aguas la cual proporciona que los vapores generados en el interior no contaminen la parte central y al condensarse caigan a los lados.

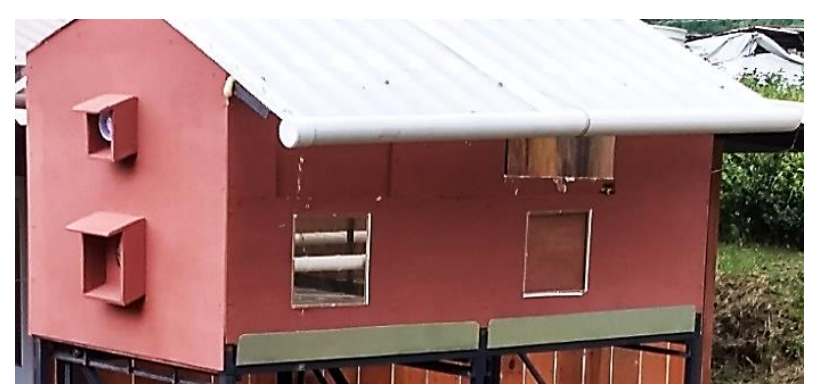

Figura 17 Estructura del tercer módulo Fuente: Elaboración propia 
El sistema cuenta con una red de suministro de oxígeno, así como extractores, dispensadores de agua y dispensadores de alimento. Para el suministro de agua se utilizó un bote con capacidad de 19 litros, el cual cuenta con un filtro que está conectado a la tubería de suministro de los bebederos para evitar que los pollos sean contaminados con algún residuo peligroso, el sistema mencionado anteriormente podemos verlo en la Figura 18.

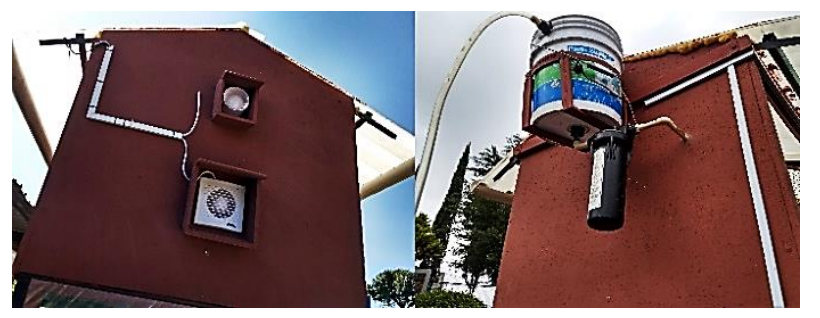

Figura 18 Sistema de extractores y dispensadores de agua Fuente: Elaboración propia

\section{Resultados}

Una vez que se ensamblaron los tres módulos se obtiene un sistema de producción que garantiza el suministro continuo de alimentos inocuos y nutritivos en tres ejes principales, producción de aves de corral, peces y hortalizas, véase Figura 19.

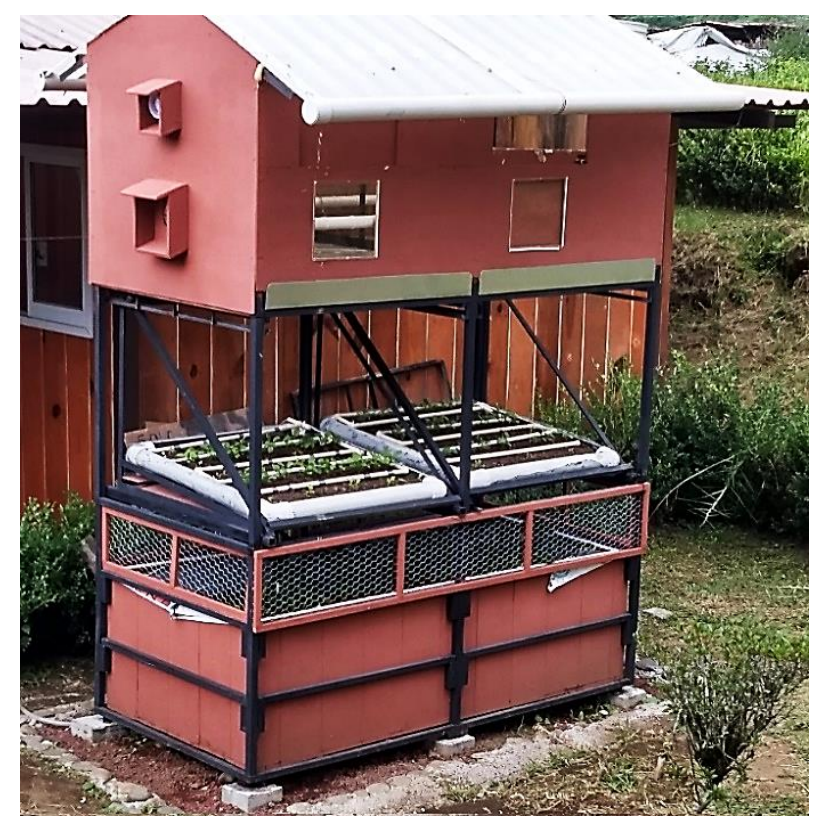

Figura 19 Prototipo terminado

Fuente: Elaboración propia
Con los sistemas en funcionamiento, se procedió a calificar los resultados obtenidos, en el caso del tercer módulo se observó que las aves de corral producen demasiado desechos, por ende, los contenedores encargados de recolectar se llenaron rápidamente, este es un excelente fertilizante comúnmente llamado gallinaza, por lo cual se optó por hacer uso de un recipiente más para su depósito para la elaboración de composta, como se muestra en la Figura 20. En los dispensadores de agua son llenados manualmente y al ser una tarea manual realizada constantemente se propone un sistema de llenado automático de los recipientes.

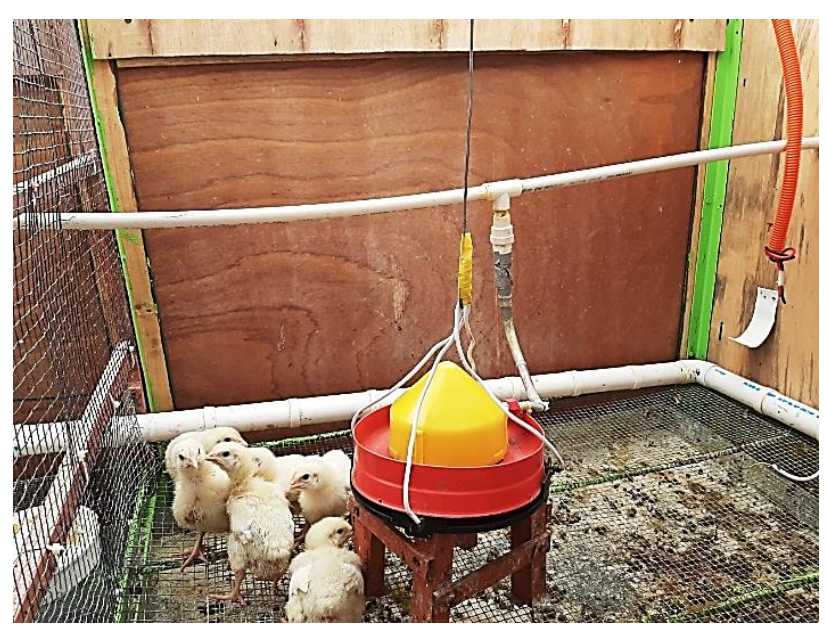

Figura 20 Contenedor para la recolección de los desechos de los pollos

Fuente: Elaboración propia

Para la producción de hortalizas se observó que algunos productos no alcanzaron un tamaño de crecimiento apropiado, debido a que no obtuvieron suficiente energía solar como se muestra en la Figura 21, por lo cual se propone un diseñó de instalación de lámparas de crecimiento, otro de los factores que determinaron que el producto no lograra un desarrollo apropiado, fue que contaban con una capa de suelo demasiado delgada, por lo cual se decidió aumentar el tamaño de la misma, también se añadieron unas capas de perlón y tezontle para evitar que la tierra se erosionara además se redujo el diámetro de las esperas, en la primera prueba liberaban demasiado flujo. 


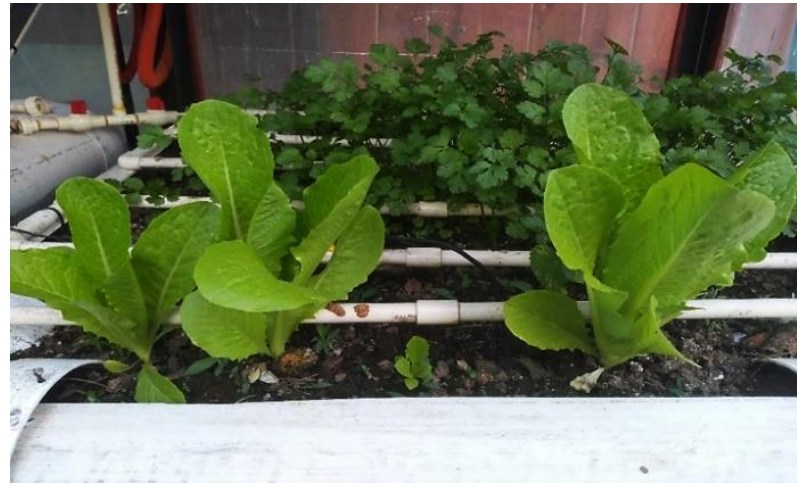

Figura 21 Producción de hortalizas

Fuente: Elaboración propia

En el caso del primer módulo correspondiente a la crianza de peces se hizo la instalación de un filtro natural, el cual se aprovechó para llenar un contenedor el cual almacena agua limpia y después es bombeada para regresar al primer contenedor y obtener mayor recirculación de agua.

Partiendo del comportamiento de las principales variables: temperatura, humedad relativa, $\mathrm{PH}, \mathrm{CO}_{2}$ y $\mathrm{NH}_{3}$ (Amoniaco) a controlar durante el año, se proponen un algoritmo de control según los valores de referencia basados en los límites permisibles para la crianza de aves, peces y cultivo de hortalizas, se concluye que los lazos de control deben ser robustos y a la vez flexibles para que el supuesto caso de cambiar de pez a cultivar, o cambio de tipo de ave u hortalizas. Finalmente, la reingeniería de proceso, generó conocimiento real de campo, lo que aporta mejoras al diseño de la segunda versión del mismo.

\section{Agradecimiento}

A la carrera de Ingeniería Mecatrónica del Tecnológico Nacional de México/Instituto Tecnológico Superior de Huauchinango por el apoyo y las facilidades en el desarrollo del proyecto, así mismo este artículo fue realizado como producto del proyecto "Diseño e integración de prototipo autosustentable vertical para crianza de peces, aves de corral y hortalizas, orientada a seguridad alimentaria en la región de la Sierra Norte de Puebla" del Cuerpo Académico "Automatización y Control de Procesos", financiado por la convocatoria Fortalecimiento de Cuerpos Académicos del PRODEP.

\section{Conclusiones}

Después de evaluar el sistema implementado e identificar las necesidades reales una vez que el sistema está funcionando, fue necesario realizar adecuaciones al diseño inicial, para no tener desviaciones mayores a las permisibles en los tres módulos de producción, según lo proyectado inicialmente respetando la normatividad de los parámetros.

En el caso de la producción de pollos, el sistema presentó una eficiencia esperada, la mortalidad de las aves se redujo de un $30 \%$ a un $10 \%$, este último porcentaje se debe a factores externos al sistema. Es de vital importancia mantener un sistema en condiciones óptimas, dado que las aves poseen un sistema inmunológico delicado, esto junto con cambios bruscos de temperatura sumada a un exceso de humedad provocaría que las aves se enfermaran y posteriormente su mortandad. Gracias al sistema de extracción de desechos que se diseñó, se utilizaron para generar composta y posteriormente utilizarla para las hortalizas como abono.

En la producción de hortalizas, la primera cosecha que se levantó fue próspera, pero el suministro de agua para obtener humedad fue sin aplicar control. Para el estanque, se detectó que los peces brincan continuamente por lo que se colocó alrededor de la misma una malla de tela para evitar que salieran del estanque.

Como resultado del proyecto, es posible concluir, que el sistema implementado nos proporciona una mejor alternativa en la producción de alimentos para autoconsumo en las viviendas rurales o urbanas.

\section{Referencias}

Alarcón Pérez, R. (2020). Desarrollo de una aplicación móvil para la comercialización de productos en el sector agrícola de Andahuaylas 2019.

Calderon-Alba, C. C., Morales-Oviedo, V., \& Perez-Alzate, Y. R. (2020). Diseño de estrategias en la empresa $\mathrm{A} \& \mathrm{C}$ para fortalecer la importación de la línea de productos fertilizantes. 
Cano, E. (2015). Huertos Familiares: Un camino hacia la Soberanía Alimentaría. Revista Pueblos y Fronteras Digital vol. 10, núm. 20, diciembre, 70-91.

CASTILLO, Gregorio, GÓMEZ, Elda, GONZAGA, Elisa y REYES, Iván, (2017). Sintonización y comparación de controladores para un aeropéndulo, Revista de Ingeniería Eléctrica, Vol. 1 No. 3, 16-26, http://www.ecorfan.org/republicofperu/research _journals/Revista_de_Ingenieria_Electrica/vol1 num3/ECORFAN_Revista_de_Ingenier\%C3\% ADa_El\%C3\%A9ctrica_VI_N3_3.pdf

Cercado Banda, M., \& Valdivia Reyes, W. B. (2020). CENTRO DE CAPACITACIÓN, INVESTIGACIÓN, PROMOCIÓN Y COMERCIALIZACIÓN AGRÍCOLA PARA CONTRARRESTAR DISFUNCIONALIDAD SECTORIAL EN PÁTAPO Y TUMÁN.

DELGADILLO GAYTAN, R. O. D. R. I. G. O. (2020). Desarrollo de una Red de Sensores para el Monitoreo en Ambiente Web de Parámetros Físico-Químicos en Invernaderos de Plantas Ornamentales (Doctoral dissertation, Instituto Tecnológico de Colima).

Durán, M. (2014). Criterios tecnológicoambientales bajo un enfoque sistémico: transferencia de tecnología química. Ingeniería Investigación y Tecnología, volumen XV (número 3), julio-septiembre, 339-350.

Fairchild Semiconductor Corporation, (2014). "MOC3010M, MOC3011M, MOC3012M, MOC3020M, MOC3021M, MOC3022M, MOC3023M. 6-Pin DIP Random-Phase Optoisolators Triac Driver Output (250/400 volt peak)". En Fairchild Semiconductor Corporation. Consultado el 24 de octubre del $2019 . \quad$ Disponible en: http://www.farnell.com/datasheets/1806097.pdf

Gregorio Castillo Quiroz, Arnulfo Cruz Garrido, Elisa Gonzaga Licona, Eugenio Luna Mejía, (2019). "Diseño e implementación de sistema de monitoreo automatizado en granja avícola", Revista de Investigación en Tecnologías de la Investigación (RITI), ISSN 2387-0893, 7 (14), Pp31-42. doi:
Johana Andrea Ciro-Galeano, Mateo Itza-Ortiz. (ABRIL de 2015). Parámetros Productivos. Recuperado el 08 de septiembre de 2019, de Parámetros Productivos: https://www.researchgate.net/publication/30835 6178_PARAMETROS_PRODUCTIVOS

Modificación de la Norma Oficial Mexicana NOM-127-SSA1-1994: Salud ambiental, agua para uso y consumo humano-Límites permisibles de calidad y tratamientos a que debe someterse el agua para su potabilización.

NMX-FF-080-SCFI-2006. Productos avícolas Carne de pollo de engorda en canal y en piezas Clasificación.

Norma Oficial Mexicana NOM-061-ZOO-1999, Especificaciones zoosanitarias de los productos alimenticios para consumo animal. Secretaría de Agricultura, Ganadería y Desarrollo Rural.

Las Naciones Unidas, (2018). Objetivos de desarrollo sostenible. Recuperado el 25 de enero de 2020 de:

https://www.un.org/sustainabledevelopment/es/ hunger/

Lopera, P. (2017). Medición de Parámetros Productivos en Avicultura. La revista global de avicultura, 23-26. Obtenido de Avicultura en producción ecológica:

https://avicultura.info/medicion-de-parametrosproductivos-en-avicultura/

Promueve Hidroponía. (14 de AGOSTO de 2014). Acuaponía más que un simple cultivo. Recuperado el 08 de septiembre de 2019, de Acuaponía más que un simple cultivo: https://conocehidroponiapromuevehidroponia.w ordpress.com/2014/08/14/acuaponia-mas-queun-simple-cultivo/

Ramírez Acevedo, A. F. (2020). C-BIOS empaque: Desarrollo de un empaque para el transporte y protección de plántulas en el plantuario del centro de Bio-Sistemas de la Universidad Jorge Tadeo Lozano.

WeatherSpark. (2018). El clima promedio en Puebla. Recuperado el 25 de enero de 2020 de: https://es.weatherspark.com/y/2214/Climapromedio-en-Puebla-M\%C3\%A9xico-durante-todoel-a\%C3\%B1o\#Sections-Clouds 